\title{
Asthma Simulated Patient Simulation Team Learning Experience using Hybrid Model Method
}

\author{
Younsook $\mathrm{Ju}^{1}$ and MuyeongSeak Yang ${ }^{2}$ \\ ${ }^{1}$ Assistant Professor, Department of Nursing, Hanil University, South Korea \\ ${ }^{2}$ Associate Professor, Department of Nursing, Jesus University, South Korea \\ ${ }^{1}$ angelqueen@hanil.ac.kr, ${ }^{2}$ medicms@jesus.ac.kr
}

\begin{abstract}
This study attempted to learn about students' experiences in facing problems through team-based simulation learning on asthmatic patients that nursing students can meet in clinical trials using hybrid model methods using asthma standardized patients and human patient simulators. As this study is a qualitative study to find basic data and the cultural specificity based on the learning experiences of the simulation team about the asthmatic simulated patients, we used the participation-observation method in clinical practice by studying the learning experiences of the simulation team about the asthmatic simulated patients.
\end{abstract}

Keywords: Students nurses, Simulation team learning experiences, Clinical experience

\section{Introduction}

Nursing education has been studying various practical training methods to enhance field practice and to achieve core skills in each field of practice [1][2]. Recently, various nursing teaching methods for clinical practices have been studied to enhance field practices and students' ability for nursing manual procedures according to practice fields. For the students to have the ability for the nursing practical-manual procedures, a clinical practice education is important because the students have adaptation ability related to theoretical knowledge, skill, and attitude through the clinical practice education. However, in the clinical practice fields, the students don't have many opportunities for practicing directly the nursing practicalmanual skills but just observe the nursing skills without a direct nursing intervention the students plan [3] because there are some difficulties such as lack of conversation with patents, protectors and medical team, refusal response of patients, their poor nursing skills and progressive clinical-practice situation than their theoretical knowledge [4][5]. To solve these problems and to provide students with conative learning circumstances for the basic knowledge and the clinical practical ability, a simulation nursing education is being used in the nursing education field which is similar to the clinical fields. It is reported that simulation education is, as a class process imitating real situations, useful for acquiring nursing practicalmanual skills with core fundamental nursing skills because the simulation education uses computerized human patient simulators, scenarios, and standardized patient models. It is also reported that this method provides the students with safe learning circumstances and

Article history:

Received (April 9, 2020), Review Result (May 12, 2020), Accepted (June 16, 2020) 
eliminates risks that may happen in real clinical practice. Likewise, it has a positive effect by improving the self-efficacy, learning achievement, and class satisfaction of the students [6][7][8][9]. Further, recently the Korean Accreditation Board of Nursing Education [KABONE] raised the clinical practice-time criteria from $10 \%$ (the second certification evaluation criteria) to $15 \%$ as the third certification evaluation criteria, which means that the simulation education increases. Simulation education inspires the students with critical thinking and promotes the absorption of knowledge related to conversation skills and clinical practice ability through the debriefing stage in which they debrief their feelings and interventions they planned when they realized the scenario between educators and learners [10]. Simulation education trains individuals or tea. In the case of team education, groups of 10 fewer are most effective [11]. However, simulation practice education improves the nursing practice ability, and trains teams more than individuals.

It is reported that learning based on the team improves the problem-solving skills, interpersonal skills, and learning accomplishment of the students because the students can experience a process of thinking and integration, application and evaluation of knowledge through active interaction which can happen in the clinical fields.

The learning method based on the team asks the students to change from their passive thinking to conative thinking so it is needed to carefully study how the nursing students accept the new learning methods like the simulation based on the team and individually experience the methods.

Many studies found some simulation education methods such as HPS (human patient simulator) and SP(standardized patients) which are mainly used in the learning based on the team. There is a limit to realizing the HPS but using well-trained SP patients can more realistically realize the symptom of patients than unstructured clinical learning circumstances. Through team learning, the team members can classify their roles and practice them. Also, they can study real patients and realize the symptoms and feelings like real which patients can feel. The students, who practice clinical training, stress out about responding to patients' requirements in unexpected clinical situations and experience losing their self-respect and great pressure. Especially, in case the patients severely complain of pain like dyspnea and quick reaction is needed for treatment, they can feel the great burden. Therefore, repeated simulation practice, which is similar to real clinical fields, is important to safely take care of the patients and for the students to gain confidence. For repeated practice, this school organized each team with 4-5 members. Also, each team was asked to select a standardized patent who was the same as the patient in the clinical practice field. Also, they were asked to decide their roles and to write their scenario during the adult nursing clinical practice. According to the study, the scenario makes students have critically thinking, select a proper intervention, and make a connection between the simulation experiences and the clinical practice experiences. We used the HPS when the standardized patient couldn't realize something like a real clinical patient. The scenarios, which students made, were reviewed by two adult nursing professors and two experts who had been working as pulmonologists for over 10 years. That is, this study is to understand the concrete-fresh education experiences of 4th-grade nursing students in-depth who experienced the clinical situation with standardized patients. 


\section{Design method}

\subsection{Study design and design method}

The study design uses qualitative methods and ethnography like interviews and observation to find out the basic materials of the learning experiences of the simulation team about the asthmatic simulated patients. There are many ways to study culture, but among them, the journal focuses on cultural perspectives as a qualitative research method that directly describes culture or lifestyle in society. Depending on how to view the concept of culture, or on what to focus research, the Journal of Culture and Technology is expressed in a variety of ways, such as the typical Journal of Culture and Daily Technical Research. The field study technique is used, which enters into the daily life site where human act happens. And, participation-observation and interview are the main method.

\subsection{Selecting the study participant and ethical consideration}

The object of study is all 4th grade 11 nursing students in S-myeon in Jeollabuk-do. We explained the purpose of the study and selected participants who wanted to participate. For the ethical consideration, we explained the study purpose and process before starting the study. Also, we received their agreement on anonymity after directly explaining that their interview contents wouldn't be used except for the purpose and that their materials would be used anonymously.

\subsection{Data collection}

The data collection period was from Sep, $21^{\text {st }}, 2016$ to Oct, 19th, 2016 when the materials reached their maximum for 4 weeks. Participants in the study took courses related to adult nursing, respiratory system disorder, and part nursing, and selected 11 students willing to participate in the study from among the third-year students of the department of four-year nursing who had clinical practice experience in the respiratory system ward. The recruitment began shortly after the clinical practice was completed, and after the simulation class, the participants' consent was obtained and recorded. In addition, to ensure participants' confidentiality, the computer was given a number that only researchers knew, removing information related to the participants. We conducted the depth interview once or twice The first interview was for 1 hour and the second interview was for 60 minutes focusing on the uncertain parts and deficient statements of the participants. Also, the interview was conducted in the researcher's laboratory when the participants wanted. The recording date was written and analyzed on the same data.

\subsection{Research problem and materials analysis}

For the problem of what is the team experience of using Hybrid model methods to simulate asthmatic patients? Two nursing doctors, who have experience in qualitative research, participated in the materials analysis process to reduce the subjective view, prejudice, and judgment errors of the researchers but to raise the study confidence and to match the categorized contents and related domain contents with the source materials. Also, a Korean literature major participated in the naming process and discuss the process. Finally, this study was taken counsel and appraisal from a nursing doctor. 


\section{Results}

\subsection{General feature of the participants}

The age distribution of the study is $23-24$ years old and the average age is 23.03 . The object of study is all 4th grade 11 nursing students in S-myeon in Jeollabuk-do. We explained the purpose of the study and selected participants who wanted to participate. The 4th grade is all 11 students consisting of 4 Christians and 4 religions. They are all single. The students living own house are 5 students and the students living apart from their own family are 6 students. 4 students entered the nursing university with recommendations and advice.

\subsection{Result according to the features of the participants}

As a result of the Learning experiences of the simulation team about asthmatic simulated patients (the qualitative study), 54 source materials, 10 components, 6 categories, and 4 domains were drawn, the 4 domains are Lack of knowledge, Difficulty of human relation and Critical thinking and confidence were drawn.

\subsection{Result}

As a result of the study, Lack of knowledge, Difficulty in human relations and critical thinking and confidence were drawn from the objects

\subsubsection{Lack of knowledge}

The students stated that they made many mistakes at first, but they made fewer and fewer mistakes and that they could remember things they had learned at school for a long time without forgetting them through the process of checking them again at the clinical site.

"I could know the rationale of the nursing skills that I didn't know by applying the nursing process." (\#6)

"By writing the scenarios, I could know diseases well and it was good to learn the nursing practical-manual skills through the simulation.” (\#3)

\subsubsection{Human relationship}

While participating in the education, we experienced pain and trial and error together, and through the process of repeating success and failure, we could see that fellowship was strengthened. As the process progressed, they showed consideration for each other, and it was extended to an accident to understand them by being in the position of a patient who was to be cared for.

"We became close through the group projects. Also, I thought that it's very important to figure out each feature." (\#7)

"I realized the importance of cooperation and communication in the nursing field." (\#9)

\subsubsection{Critical thinking}

Previously, nurses' nursing activities were often overlooked, but they were often overlooked, but they were carefully monitored and sometimes recognized the importance of basic nursing as they found the wrong nursing behavior.

"Because I could look at the activities of the other teams through a debriefing time, I felt lots of things differently from them and I could be developed." (\#9) 
"I could learn things that I couldn't realize through another team." (\#10)

\subsubsection{Confidence}

I had a lot of fear when I approached the patient. When Shin Hwan came from the ward, I thought, "That's how they do it." I was scared to approach the patient, but now I've seen the professor do it many times and I've tried it, so I've lost a lot of fear [11].

"My thinking skills, understanding, and consideration was improved." (\#3)

"Because I realized the scenarios that I wrote, I felt self-confidence and sense of accomplishment." (\#11)

\section{Discussion}

The objects of the study could realize various responses of the patients and the importance of communication through the simulation learning program process based on team. They also could improve their clinical nursing knowledge level and gain the confidence of the team nursing practice. This is the result of the already experienced practice and repetitive learning. Therefore, it is very important to afford the students the practice opportunity like clinical fields with the standardized patient and it can improve their practicing skills and confidence in nursing practice skills. This simulation education accords with a study that a critical thinking ability can be learned and a selection ability can be improved through simulation education.

As a result of this study, the learning experience of the simulation team about asthmatic simulated patients made the student build nursing knowledge and developed their critical thinking skills. Meanwhile, they experienced relationship difficulty when they communicated with patients or colleagues. These experienced show that students can take time for selfexamination through the learning experience. Also, they can experience the acquisition of knowledge and human relationship and they can gain self-confidence through critical thinking.

\section{Limitation point of the study}

This study was conducted on nursing students in the third year of the four-year university, and there is a limit to generalizing.

\section{References}

[1] Yoon Me-Ok and Ju Youn-Sook, "The effects of peer mentoring learnings-based preclinical OSCE program on self-confidence on core basic nursing skills and critical thinking disposition for nursing student,” Journal of Digital Convergence, vol.15, no.7, pp.285-295, (2017) DOI: 0.14400/JDC.2017.15.7.285

[2] Harden R.M. "Looking back to the future: a message for a new generation of medical educators," Medical Education, vol.45, no.8, pp.777-784, (2011) DOI: 10.1111/j.1365-2923.2011.03934.x

[3] Garrett B., MacPhee M., and Jackson C, "High-fidelity patient simulation: considerations for effective learning," Nursing Education Perspective, vol.31, no.5, pp.309-313, (2010)

[4] Bremner M.N., Aduddell K., Bennet D.n., and Van Gesst J.B, "The use of human patient simulators: Best practice with novice nursing students," Nurse Educator, vol.31, no.4, pp.170-174, (2006)

[5] Jocelyn G., Trudy H., Rolando L., and Michelle M., "Team training simulation in perioperative nursing education," The Journal of Nursing Education, vol.48, pp,388-394, (2006)

[6] Judith A. H., Janet M. P., Angela K., Karen H., Marjorie L. P., and Jennifer S. D., "Preparing nurse educators to use simulation technology: A consortium model for practice and education," Journal of Continuing Education in Nursing, vol.42, pp.496-502, (2011) DOI: 10.3928/00220124-20110502-01 
[7] Lewis D., and Ciak A., "The impact of a simulation lab experience for nursing students," Nursing Education Perspectives, vol.32, no.4, pp.256-258, (2011)

[8] Kneebone R., "Simulation in surgical training; educational issues and practical implication," Med Educ, vol.37, no.3, pp.267-275, (2003)

[9] Kim H. R., Choi E. Y., and Kang H. Y.," Simulation module development and team competency evaluation," The Korean Journal of Fundamentals of Nursing, vol.18, no.3, pp.392-400, (2011)

[10] Frengley R. W., Weller J., Weller J. M., Torrie J., Dzendrowskyj P., and Yee B., et al., "The effect of a simulation-based training intervention on the performance of established critical care unit teams," Critical Care Medicine, vol.39, no.12, pp.2605-2611, (2011)

[11] Kim Y.J. and Chin, M.H, "The lived experience of nursing students for essential nursing core skills," Qualitative Research, vol.13, no.2, pp.105-116, (2012)

\section{Authors}

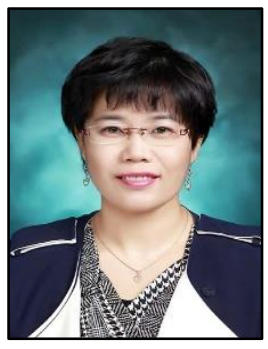

Younsook Ju, RN, RhD

Assistant Professor

College of Nursing, Hanil University

[55359] 726-15, Waemok-ro, Sanggwan-myeon, Wanju_Gun, Jeollabukdo, Republic of Korea

Tel: +82-63-230-5676

E-mail: angelqueen@hanil.ac.kr

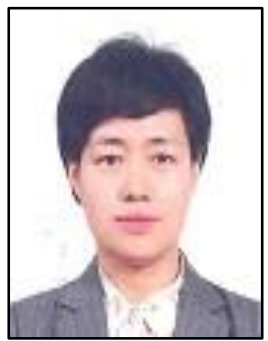

\section{Muyeong s eak Yang, RN, RhD}

Associate Professor

College of Nursing, Jesus University

[54989] 383, Seowon-ro, Wansan-gu, Jeonju-si, Jeollabuk-do, Republic of Korea

Tel: +82-63-230-7758

E-mail:medicms@jesus.ac.kr 